\title{
DiVA
}

Downloaded from the open archive of Mid Sweden University DIVA.

This is the accepted version of a paper published in IECON 2019 - 45th Annual Conference of the IEEE Industrial Electronics Society. See full citation below.

Barg, Sobhi; Alam, Farhan; Bertilsson, Kent " Modeling of the Geometry Effect on the Core Loss and Verification with a Measurement Technique Based on the Seebeck Effect and FEA," IECON 2019 45th Annual Conference of the IEEE Industrial Electronics Society, pp.1832-1837, 14-17 October 2019 10.1109/IECON.2019.8927344

(c) 2019 IEEE. Personal use of this material is permitted. Permission from IEEE must be obtained for all other uses, in any current or future media, including reprinting/republishing this material for advertising or promotional purposes, creating new collective works, for resale or redistribution to servers or lists, or reuse of any copyrighted component of this work in other works. 


\section{Modeling of the Geometry Effect on the Core Loss and Verification with a Measurement Technique Based on the Seebeck Effect and FEA}

\author{
Sobhi Barg \\ Department of Electronics Design \\ Mid Sweden University \\ Sundsvall Sweden \\ sobhi.barg@miun.se
}

\author{
Farhan Alam \\ Department of Electronics Design \\ Mid Sweden University \\ Sundsvall Sweden \\ FarhanMuhammad.Alam@miun.se
}

\author{
Kent Bertilsson \\ Department of Electronics Design \\ Mid Sweden University \\ Sundsvall Sweden \\ kent.bertilsson@miun.se
}

\begin{abstract}
In this paper, the effect of the core geometry of nontoroidal magnetic cores on the magnetic loss is investigated. A frequency dependent core material-geometry loss factor is developed. This factor is function of the change in the non-toroidal core section and the Steinmetz parameter " $\beta$ ". In addition, the temperature effect is included in the developed loss model for wide range of frequency and magnetic flux density. The model is applied for ER core and 3C92 ferrite material. The core loss measurements are performed using a Peltier cell. The principle of operation of the Peltier cell is based on the Seebeck effect, which convert the heat flow due to the temperature difference into electric power. The calibration of the Peltier cell is validated with a resistive load and a relative error lower than $1 \%$ is achieved. The accuracy of the developed model is assessed with FEA and the experimental results. A maximum error of $10 \%$ is registered of the developed core loss model.
\end{abstract}

Keywords—core loss, core geometry effect, Peltier cell, Seebeck effect, FEA.

\section{INTRODUCTION}

Magnetic core loss has a non-linear variation with respect to the frequency, the material, the magnetic field and the temperature. This makes its calculation one of the most difficult tasks in the design of high frequency magnetic devices. Empirical models, based on the Steinmetz equation (SE), are more preferable for power electronics designers than the separation loss methods or the hysteresis-based-models because of its easiness and accuracy [1]. These two features are achievable because the Steinmetz parameters can be directly obtained from the loss measurements given in the datasheets. The Steinmetz-based-models are mainly the generalized Steinmetz equation, the modified Steinmetz equation, the improved generalized Steinmetz equation, the Improved $^{2}$ Steinmetz equation and the Improved Steinmetz equation [2-8]. Nevertheless, these methods has only investigated the core loss under different voltage waveforms regardless the effect of the core geometry. However, this later is also one crucial factor that has a significant effect on the core loss. As an example, measurements performed on powder material shows that the loss of $\mathrm{E}$ cores is about $50 \%$ lower than the loss of an equivalent toroid core [9]. Despite the significant impact of the core geometry on core loss, still we do not find any research that investigated and characterized its effect. Existing empirical models such as the Steinmetz equation and its derivatives are only adequate for toroidal core because they are based on the manufacturer's data, which are commonly performed on toroid cores. Eventually, the use of such Steinmetz-based-model to predict core loss in the design process of non-toroidal cores can lead to significant errors.

As previously mentioned, the empirical methods are based on the experimental loss measurements. However, measuring the magnetic loss at high frequency with high accuracy is a demanding and difficult task, which requires a lot of efforts and expertise. Two popular methods are proposed in the literature: the calorimetric methods and the electrical methods [10] and [19]. The second technique is easy to implement, as it only needs to measure the current and the voltage across the DUT. However, due to the limitations of the measurement instruments (bandwidth of the current sensor and oscilloscope, phase shift, probes delays ...), the accuracy might be affected especially at high frequency where the $\mathrm{di} / \mathrm{dt}$ and $\mathrm{dV} / \mathrm{dt}$ can have a great impact on the digital circuits [10-11].

On the other hand, the calorimetric technique is time demanding but it can have good accuracy since all the power loss is dissipated as heat and can be measured directly. The accuracy can reach very acceptable value by minimizing the leakage heat. The power loss can be known by measuring the temperature difference and the flow rate of the cooler (water or air). The temperature and the flow rate of the cooler needs to be controlled to compensate its effect of the power loss measurement, which complicates the design of the measurement setup. In addition to that, this technique is costly and time consuming [12-13].

One quick and easy calorimetric measurement setup, based on the Seebeck and Peltier effects, is also presented in the literature. It was used for the measurement of magneto-caloric effect and electronic devices loss [14-18]. A thermoelectric device called Peltier cell (TEC) is used to generate electric power through heat flow due to the difference in temperature. The structure of this device is two ceramic plates connected number or series connected thermoelectric elements. Principally, the Peltier cell has two dual functions: (1) sensor function based on the Seebeck effect and (2) actuator function, based on the Peltier effect. The main advantages of this calorimetric measurement method are summarized as follow. First, it has high accurate readings 
because the electric signal is much greater than using a thermal shunt. Eventually, it makes it suitable for low power loss measurement. Furthermore, it has a low thermal resistance to a heat sink and thus little heating of the DUT. In addition to that, the experimental implementation is quite easy because it does not need control and adjustment issues of the cooler heat flow in the conventional calorimetric technique. Finally, it is not time consuming and not expensive compared to other methods.

In this work, the Seebeck effect is used to measure the HF core loss of ferrite non-toroidal core. The Seebeck voltage measured across the Peltier cell due to the temperature gradient between the ceramic plates can give accurate prediction of the core loss. The temperature gradient is generated by connecting the one ceramic plate to the magnetic core and the second plate to the heat sink. More details about the characterization of this method for core loss measurement is given in section III.

In conclusion, the design of a simple and accurate core loss model for non-toroidal cores to include the effect of the core geometry and the development of a simple and quick core loss measurement technique is of great importance and this is the main objective of this research.

The main contributions of this paper are:

1. To develop an accurate and straightforward core loss model that includes the effect of the core geometry.

2. To present a novel core loss measurement technique based on the Seebeck effect.

3. To verify the accuracy of the developed core loss model with $\mathrm{FE}$ analysis and measurement results.

The paper is structured as follows. In the second section, a straightforward and accurate core loss model is developed to include the effect of the core geometry and the temperature. The experimental setup of the Peltier measurement technique is described in section III. The FEA analysis of the core geometry effect on the core loss is presented in section IV. The discussion and the evaluation of the obtained results are presented in section V. Finally, the conclusion is summarized in section VI.

\section{CORE LOSS MODEL INCLUDING THE CORE GEOMETRY EFFECT}

Magnetic core loss depends on four main variables: the magnetic flux density, the frequency, the material and the temperature. The frequency and the material are fixed variables, which are chosen by the designer for a given application. The temperature depends on many factors such as the material, the temperature rise, which in turn depends on the power and the core volume and the external temperature. The most crucial variable is the magnetic flux density because it depends on the temperature in addition to the applied voltage and the core section. This later is uniform for toroid core, however, it is not the case for non-toroidal cores such as the EE, ETD, PQ, ER...cores. As a result, the intensity of the magnetic flux density changes within the core due to the change of the section. This makes the core loss within the core inhomogeneous.

One common way to predict the magnetic flux density is to calculate its average value using the mean core section usually known as the effective section. The idea can have accurate results in condition that the effective cross section is equal to the minimum section in the core. However, the accuracy degrades when the previous condition is not satisfied. This is because the averaging calculation of $\mathrm{B}$ for core loss can lead to wrong loss prediction since the core loss is a non-linear phenomenon. In the following, we present a method to characterize the effect of the core geometry on the core loss.

\section{A. Core material-geometry loss factor modeling}

A typical non-toroidal core can be divided into three different parts: the center leg part, the base part and the outer legs part. The section of these three parts are usually slightly different which significantly affects the homogeneity of the magnetic flux density within the core. This study deals with the case of ER32/6/25 core (Fig.1 and Tab.1) where the ratio between the effective and minimum core sections is considerable [21].

The superposition technique is proposed to accurately calculate the core loss for non-toroidal cores. Based on this method, a core geometry loss factor is determined to better characterize the core loss.

The core loss of the base, the outer legs and the center legs are respectively given by the model developed in [8]:

$\left\{\begin{array}{l}P_{c 1}=\frac{\pi}{4} k f^{\alpha} B_{m 1}{ }^{\beta} V_{1} \\ P_{c 2}=\frac{\pi}{4} k f^{\alpha} B_{m 2}{ }^{\beta} V_{2} \\ P_{c 3}=\frac{\pi}{4} k f^{\alpha} B_{m 3}^{\beta} V_{3}\end{array}\right.$

The mean magnetic flux density is by definition as follows.

$B_{e}=\frac{V \Delta T}{N f A_{e}}$

$\mathrm{B}_{\mathrm{m} 1}, \mathrm{~B}_{\mathrm{m} 2}$ and $\mathrm{B}_{\mathrm{m} 3}$ can be expressed as a function of $\mathrm{B}_{\mathrm{e}}$.

$\left\{\begin{array}{l}B_{m 1}=B_{e} \frac{A_{e}}{A_{1}} \\ B_{m 2}=B_{e} \frac{A_{e}}{A_{2}} \\ B_{m 3}=B_{e} \frac{A_{e}}{A_{3}}\end{array}\right.$

The total core loss is the sum of the losses within the different parts.

$P_{c t}=P_{c 1}+P_{c 2}+P_{c 3}$

Replacing (3) into (1) and (1) into (4), we get the core loss per unit volume as follows:

$P_{c t u}=\frac{\pi}{4} k f^{\alpha} B_{e}^{\beta} C_{g e}$

$C_{g e}=A_{e}^{\beta}\left(\frac{1}{A_{1}{ }^{\beta}} \frac{V_{1}}{V_{t}}+\frac{1}{A_{2}{ }^{\beta}} \frac{V_{2}}{V_{t}}+\frac{1}{A_{3} \beta} \frac{V_{3}}{V_{t}}\right)$

Where $C_{g e}$ is called the core material-geometry loss factor. It depends on the sections ratio, the volume ratio and the 
Steinmetz parameter $\beta$ which in turn depends on the material and the frequency. $\mathrm{V}_{\mathrm{t}}$ is the total core volume, $\mathrm{V}_{1}, \mathrm{~V}_{2}$ and $\mathrm{V}_{3}$ are respectively the volumes of part I, part II and part III. Fig.2 shows the variation of the Steinmetz parameters and $\mathrm{C}_{\mathrm{ge}}$ with respect to the frequency for ER32/6/25 core made of $3 \mathrm{C} 92$ ferrite material. $C_{g e}$ factor can vary by $2 \%$ in the range of $[25-$ $500] \mathrm{kHz}$. Fig. 3 shows how accurate the Steinmetz parameters by comparing the specific core loss given in the datasheet and the core loss calculated using Steinmetz equation.

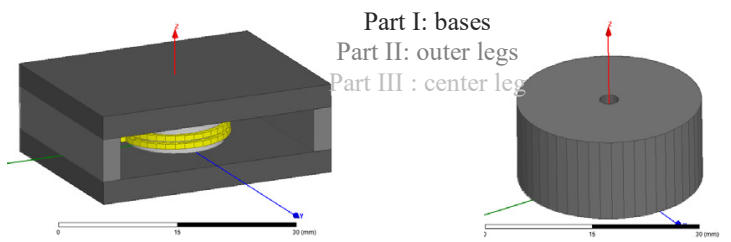

Fig. 1. Designed ER32/6/25 core and its equivalent toroid core

TABLE I. MAGNETIC CORE SPECIFICATIONS

\begin{tabular}{|l|c|}
\hline Core & ER32/6/25 \\
\hline material & 3 C92 \\
\hline Effective Volume $\left(\mathrm{mm}^{3}\right)$ & 5400 \\
\hline Effective section $\left(\mathrm{mm}^{2}\right)$ & 141 \\
\hline Minimum section $\left(\mathrm{mm}^{2}\right)$ & 121 \\
\hline Effective length $(\mathrm{mm})$ & 45.7 \\
\hline
\end{tabular}
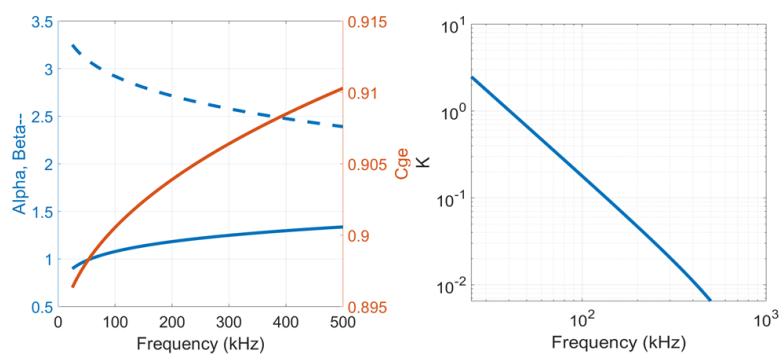

Fig. 2. Steinmtez parameters and Cge models as a function of the frequency for ER $32 / 5 / 25$ core made of 3 C 92 ferrite material

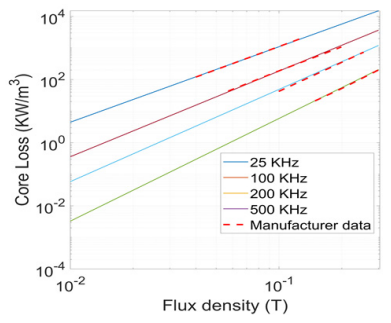

Fig. 3. Derived core loss model and core loss curves from datasheet $\left(T=100^{\circ} \mathrm{C}\right)$

\section{B. Thermal modeling}

As formerly, mentioned, the temperature has a significant effect on the core loss (Fig.4) and its consideration on the core loss modeling is inevitable [20]. Modeling of a non-linear phenomenon with three variables $(f, B, T)$ is quite difficult problem especially when the experimental data are not sufficient [19]. In our case problem, the specific core loss of the 3C92 material for wide ranges of flux density and frequency are only given for $100^{\circ} \mathrm{C}$ while our measurements are performed at $25^{\circ} \mathrm{C}$ [19]. Fortunately, some thermal core loss data are given for particular $B$ and $f$ (Fig.4). In order to adapt the loss data of the manufacturer with our measurements and to evaluate the developed model, it is necessary to figure out the temperature effect on the core loss. The modeling process is achieved as follows. As it can be seen in Fig.4, for a given $(f, B)$, the core loss can be modeled by a second order polynomial with respect to the temperature in the range $[0-100]^{\circ} \mathrm{C}$. It can also be expressed as a function of the Improved Steinmetz equation and a normalized function $\mathrm{g}(T)[8]$.

$\left.P_{c t u}(T)\right|_{f, B}=\left.\frac{\pi}{4} k f^{\alpha} B_{m n}{ }^{\beta} C_{g e}\right|_{100^{\circ} \mathrm{C}} g(T)$

$g(T)=a T^{2}+b T+c$

(a, b and c) are parameters derived by curve fitting and $\mathrm{g}(T)$ can be defined as the normalized temperature's loss effect at a given $(f, B)$.

For a given temperature, $\mathrm{g}$ can be expressed as a function of $B$. For example, at $f=100 \mathrm{kHz}$ and $T=25^{\circ} \mathrm{C}$, its expression is.

$g\left(25^{\circ} \mathrm{C}\right)=x B+y$

Hence, the expression of $\mathrm{g}$ as a function of $\mathrm{T}$ and $\mathrm{B}$ is:

$g(\mathrm{~T}, \mathrm{~B})=x(T) B+y(\mathrm{~T})$

Then, the expression of the core loss for a given frequency is:

$\left.P_{c t u}(T, B)\right|_{f}=\left.\left.\frac{\pi}{4} k f^{\alpha} B_{m n}{ }^{\beta} C_{g e}\right|_{100^{\circ} \mathrm{C}} g(T, B)\right|_{f}$

We define the normalized function of $\mathrm{g}(\mathrm{T}, \mathrm{B})$ by:

$g_{n}(T, B)=\frac{g(T, B)}{g(T, 0.1)}$

The same approach is implemented to determine the effect of the temperature at different frequencies and at a given $\mathrm{B}(0.1$ T).

$$
\begin{aligned}
& \left.P_{c t u}(T, f)\right|_{B}=\left.\left.\frac{\pi}{4} k f^{\alpha} B_{m n}{ }^{\beta} C_{g e}\right|_{100^{\circ} \mathrm{C}} f(T, f)\right|_{B=0.1} \\
& f(\mathrm{~T}, \mathrm{~B})=r(T) f+s(\mathrm{~T})
\end{aligned}
$$

$\mathrm{r}(\mathrm{T}), \mathrm{s}(\mathrm{T}), \mathrm{x}(\mathrm{T})$ and $\mathrm{y}(\mathrm{T})$ are second order polynomials. Finally, we get the thermal core loss model:

$P_{c t u}=\left.\frac{\pi}{4} k f^{\alpha} B_{m n}{ }^{\beta} C_{g e}\right|_{100^{\circ} \mathrm{C}} f(T, f) g_{n}(T, B)$ 


\section{DESCRIPTION OF THE EXPERIMENTAL SETUP}

In this paper, the Peltier cell TEC1-12706 is used to measure the core loss of a ferrite magnetic core excited with a triangular flux excitation [22]. The details of the tested magnetic core are summarized in Tab.1. A Half bridge circuit is built to generate the required flux waveform (Fig.5). The switching frequency and the PWM signals are digitally controlled using Microship dsPIC. The magnetic flux density within the core is calculated through the measured voltage across the secondary winding (one turn) to avoid the drop voltage in the primary resistance due to the magnetizing current. The current absorbed by the transformer is also measured using a current probe.

The Peltier cell is placed between the magnetic core and the heat sink in order to generate the temperature gradient, which in turn will generate the Seebeck voltage. Insulation material is used to isolate the magnetic core and the Peltier cell from the air to allow the dissipation of all the heat through the Peltier cell. Thus, the only mechanism of heat transfer existing in our test setup is the conduction heat transfer from the magnetic core through the Peltier cell to the heat sink. The conduction through the isolation material is considered to be negligible. To verify its influence the accuracy of the test setup have been characterized below.

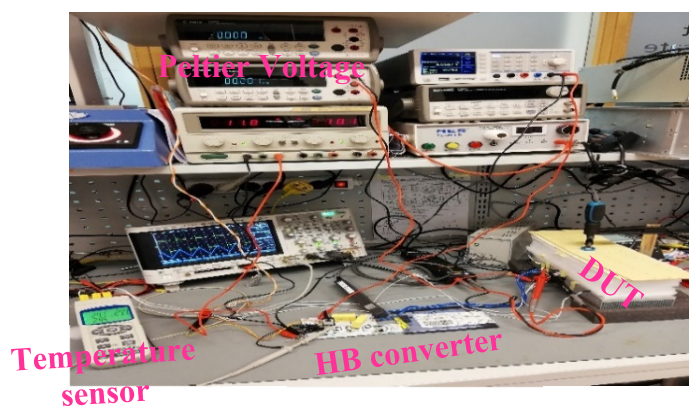

Fig. 5. Test setup of the core loss measurement

The temperature of the upper and the lower ceramic plates of the Peltier cell are equal to the temperature of the magnetic core and the temperature of the heat sink respectively. The use of the TEC cell for core loss measurements needs two main steps: the calibration and the determination of the time constant.

Calibration: the calibration of the Peltier cells aims to determine the relationship between the power loss and the voltage of the Peltier cells. This step is performed using a resistance using a direct current to ensure accurate readings. The Seebeck voltage, the resistor voltage and the resistor current are measured using a high-resolution digital multi-meter (HCM8012).

The measured data are shown in Fig.6-a. The Peltier voltage shows a linear variation with respect to the input power P. Eq (16) gives the fitting equation of this variation. The accuracy of the fitting equation is enough for the power range of $[10 \mathrm{~mW}$ $7 \mathrm{~W}]$. The maximum relative error between the fitting function and the measurement data is $1 \%$ at $10 \mathrm{~mW}$. In the range of $[0.1 \mathrm{~W}-4 \mathrm{~W}]$ is lower than $0.1 \%$ which satisfies the core loss measurement at high efficiency (Fig.6-b).

$$
P=15.92 V_{p l}
$$

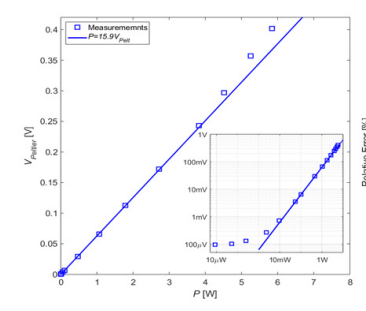

(a)

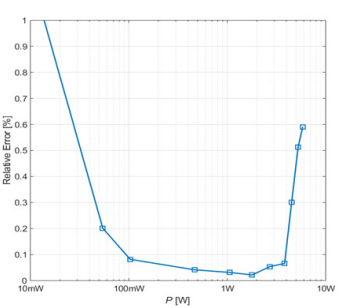

(b)
Fig. 6. Variation of the Peltier voltage (a) and the relative error (b) with respect to $\mathrm{P}$

Time constant: the second step consist in determining the time constant needed to reach the thermal equilibrium of the DUT. The test is performed for different voltages. Fig.7 shows the variation of the normalized Peltier voltage for different inputs power. It is clear that the time needed for the DUT to reach its thermal equilibrium is independent on the input power. The variation of the normalized Peltier voltage can be written as given in $\mathrm{Eq}(17)$ :

$V_{p l N}=1-e^{-\left(\frac{t-t_{d}}{\tau}\right)}$

Where $\tau(32 \mathrm{~s})$ is the time constant and $t_{d}(3 s)$ is a time delay, which physically represents the time needed for the DUT to react from the heat source.

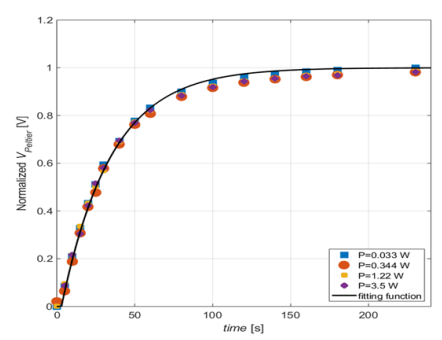

Fig. 7. Peltier voltage variation with respect to time

\section{CORE LOSS ANALYSIS WITH FEA}

FEA is a popular approach when dealing with the design of electromagnetic devices. In this work, it is used to calculate the core loss and in particular to investigate the core materialgeometry effect on the core loss for non-toroidal magnetic cores.

The transient solver in Maxwell Ansys software is selected for this objective. The excitation source is a square voltage source and the magnitude of the voltage source is swept to generate the required magnetic flux density. The process is repeated for 100 $\mathrm{kHz}$ and $200 \mathrm{kHz}$. The investigated magnetic core is ER32/5/6/25 from ferroxcube. This core has an effective core section of $141 \mathrm{~mm}^{2}$. The ratio between the effective core section and the maximum and minimum sections are 0.89 and 1.16 respectively. The maximum and the minimum sections correspond to the sections of Part I and part III respectively. The magnetic material is 3C92 and its characteristics are assigned to the designed core. The characteristics includes the permeability, the Steinmetz parameters, mass density, etc (Fig.2). An equivalent toroid core of same volume to ER32/5/25 
was also designed for loss comparison. Obviously, the section of the equivalent toroid core is equal to the effective section of the ER core. Thereafter, a meshing is created and the simulation is run. The magnitude of the magnetic flux density across the three parts of the core is presented on the $\mathrm{XZ}$ and $\mathrm{YZ}$ planes as given in Fig.8. The flux density on the inner surface of part $I$ is shown on the XY plan. For all cases, it is clear that most of the area in parts II and III are subjected to a flux density higher than the effective flux density, however, a significant portion of part I is subjected to a flux density lower than the effective flux density. This is because the section of part I is bigger than the effective core section, which is not the case for sections II and III.

In comparison with the equivalent toroid core, it can be seen that the maximum magnitude of the magnetic flux density around the inner diameter is much higher than the one for the simulated ER core. About one-fifth of the area is subjected to a $B$ higher than the effective magnetic flux density $\left(B_{e}\right)$ for the equivalent toroid; however, this area is much lower for the ER core (Fig.9).

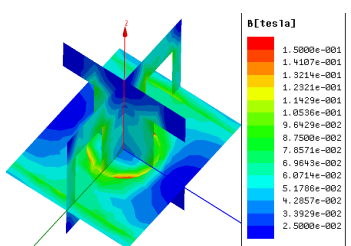

(a) $\mathrm{B}_{\mathrm{e}}=0.05 \mathrm{~T}$

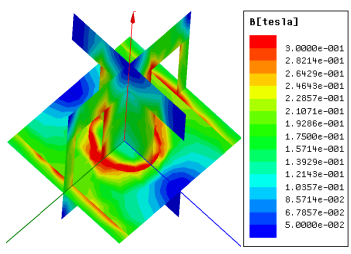

(c) $\mathrm{B}_{\mathrm{e}}=0.15 \mathrm{~T}$

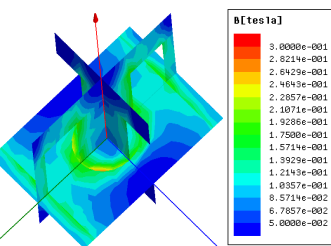

(b) $\mathrm{B}_{\mathrm{e}}=0.1 \mathrm{~T}$

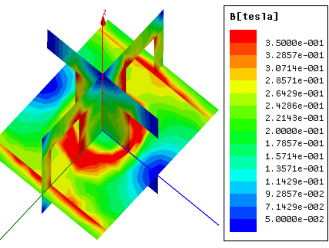

(d) $\mathrm{B}_{\mathrm{e}}=0.2 \mathrm{~T}$
Fig. 8. Magnitude of the simulated B across XY, XZ, YZ planes of ER32/6/25 core, $\mathrm{f}=200 \mathrm{kHz}$

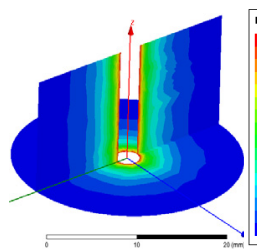

(a) $\mathrm{B}_{\mathrm{e}}=0.05 \mathrm{~T}$

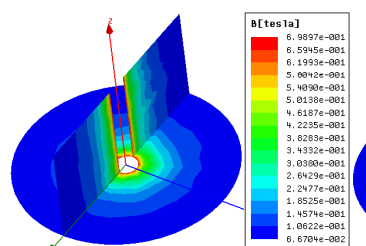

(c) $\mathrm{B}_{\mathrm{e}}=0.15 \mathrm{~T}$

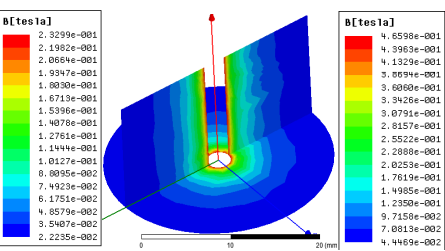

(b) $\mathrm{B}_{\mathrm{e}}=0.1 \mathrm{~T}$

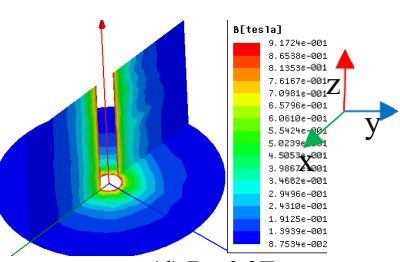

(d) $\mathrm{B}_{\mathrm{e}}=0.2 \mathrm{~T}$

Fig. 9. Magnitude of the simulated $\mathrm{B}$ across $\mathrm{XY}, \mathrm{XZ}$ planes of the equivalen toroid core, $\mathrm{f}=200 \mathrm{kHz}$

Fig.10 depicts the core loss density of the ER and the equivalent toroid cores. Since part II and Part II have lower section than part I, their loss density is obviously higher as it can be seen in Fig.10-a. The equivalent toroid core shows a higher loss density compared to the ER core. It should be noted that the core loss depends on the magnetic flux distribution. The loss density tends to increase around the edge and on the main flux path. This issue is not investigated in our paper and will be covered in a future work.

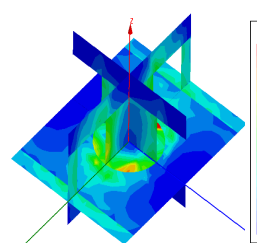

(a) ER core

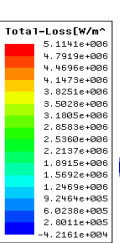

(20)

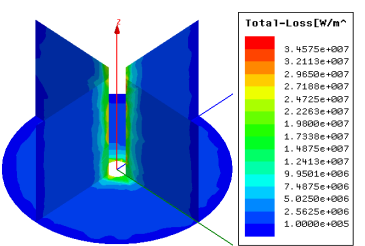

(b) Equivalent toroid
Fig. 10. Simultaed core loss density, $\mathrm{B}_{\mathrm{e}}=0.2 \mathrm{~T}, \mathrm{f}=200 \mathrm{kHz}$

\section{DISCUSSIONS OF THE RESULTS}

Fig.11 shows a comparison between the theoretical and the simulated core material-geometry loss factor. For $f=100 \mathrm{kHz}$, $C_{g e}$ is higher than $C_{g e F E A}$ however it is the opposite for $200 \mathrm{kHz}$. The difference could be to the effect of the flux distribution through the core which is not taken into account.
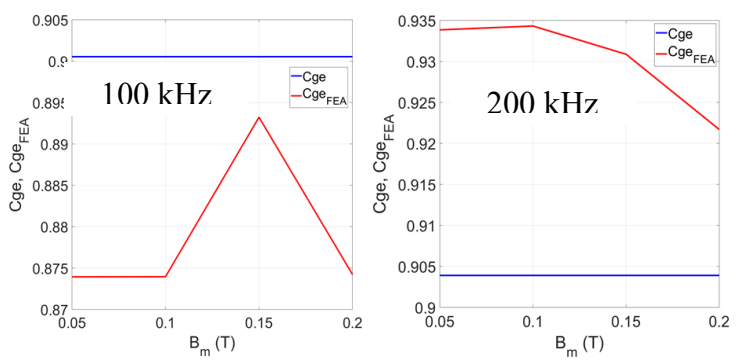

Fig. 11. Variation of the calculated and the simulated Cge with respect to $\mathrm{B}$

Fig. 12 shows the evolution of the developed core loss model and the losses obtained from FEA and measurements with respect to B. The accuracy of the developed model can be evaluated by calculating the relative error with reference to the FEA and the measurement results. The maximum registered error is around $10 \%$ in comparison to the measurements (Fig.14). From Fig.13, it can be concluded that the accuracy can be increased by considering the effect of the core geometry. In fact, the core material-geometry factor has reduced the loss error without $\mathrm{C}_{\mathrm{ge}}$ by more than $10 \%$.

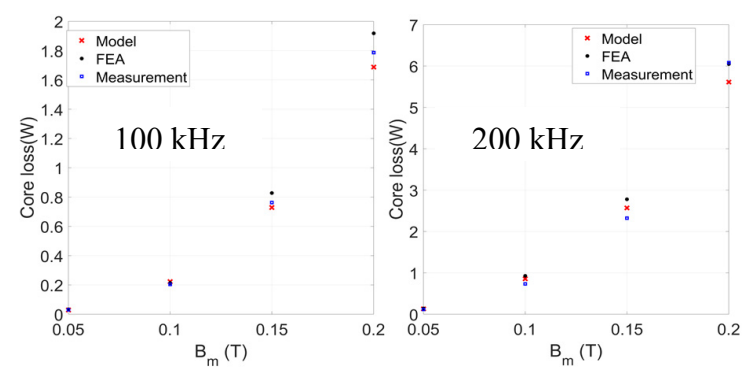

Fig. 12. Developed model, FEA and measurements losses comparison of ER32/6/25 


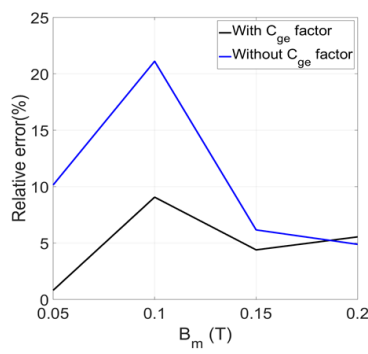

(a) $\mathrm{f}=100 \mathrm{kHz}$

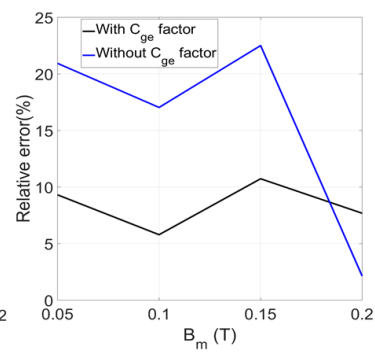

(b) $\mathrm{f}=200 \mathrm{kHz}$
Fig. 13. Relative errors of the developed model with and without the core geometry factor

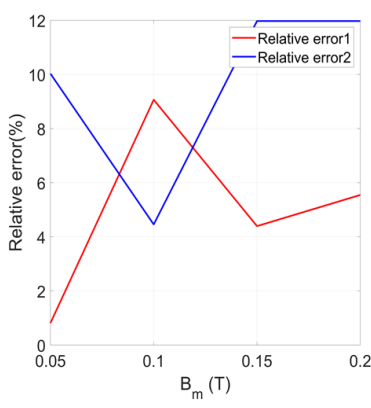

(a) $\mathrm{f}=100 \mathrm{kHz}$

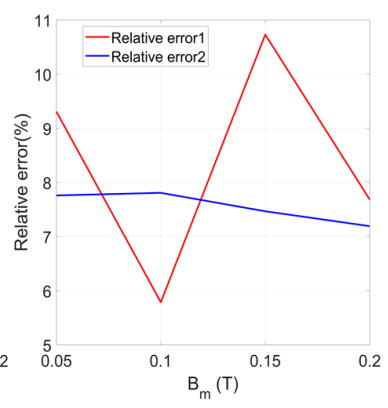

(b) $\mathrm{f}=200 \mathrm{kHz}$
Fig. 14. Relative error of the developed model witt respect to FEA (error2) and measurements (error1)

\section{CONCLUSION}

This paper has proposed a formula to compute the core loss due to the effect of the core geometry in non-toroidal cores. The core was divided into discrete pieces and the core loss of each is calculated based on the Improved Steinmetz equation function of the effective flux density and the section ratio. Then, the discrete magnetic losses are sum up and the formula of the core geometry effect is derived. The formula is a non-linear function because it is not only function of the core dimensions but also on the Steinmetz parameter " $\beta$ " which in turn depends on the magnetic material and the frequency. In addition, a new measurement technique based on the Seebeck effect is presented to measure the core loss and verify the developed formula. FE analysis was also performed to better evaluate the theoretical model. The obtained results from the experiments and the FEA shows an acceptable agreement with the developed core loss model. Despite that, the maximum registered relative error has reached $10 \%$, the developed model shows much better accuracy than the conventional Steinmetz-based models presented in the literature.

\section{REFERENCES}

[1] C. P. Steinmetz, "On the law of hysteresis," AIEE Trans., vol. 9, pp. 3-64, 1892. Reprinted under the title "A Steinmetz contribution to the ac power revolution," Introduction by J. E. Brittain. Proc. IEEE, vol. 72, no. 2, pp. 196-221, 1984.

[2] E. Agheb and H K. Høidalen, "Modification of empirical core loss calculation methods including flux distribution," IET Elect. Power Appl., vol. 7, pp. 381-390, 2013
[3] J. Reinert, A. Brockmeyer, and R.W. De Doncker, "Calculation of losses in ferro- and ferrimagnetic materials based on the modified Steinmetz equation," in Proc. 34th Annu. Meet. IEEE Ind. Appl. Soc., 1999, vol. 3, pp. 2087-2092

[4] J. Reinert, A. Brockmeyer, and R.W. De Doncker, "Calculation of losses in ferro- and ferrimagnetic materials based on the modified Steinmetz equation," IEEE Trans. Ind. Appl., vol. 37, no. 4, pp. 1055-1061, Jul./Aug. 2001.

[5] J. Li, T. Abdallah, and C. Sullivan, "Improved calculation of core loss with nonsinusoidal waveforms," in Proc. IEEE Ind. Appl. Soc. Annu. Meet., Oct. 2001, pp. 2203-2210.

[6] J. Muhlethaler, J. Bielay. J. W. Kolar, and A. Ecklebez, "Improved core loss calculation for magnetic components employed in power electronic systems," IEEE Trans. Power Electron., vol. 27, no. 2, pp. 964-973, Feb. 2012.

[7] C. R. Sullivan, J. H. Harris, and E. Herbert, "Core loss predictions for general PWM waveforms from a simplified set of measured data," in Proc. 25th Annu. IEEE Appl. Power Electron. Conf. Expo., 2010, pp. 10481055 .

[8] S. Barg, K. Ammous, H. Mejebri, A. Ammous, “An Improved Empirical Formulation for Magnetic Core Losses Estimation Under Nonsinusoidal Induction", IEEE Transactions on Power Electronics,vol. 32 , No. 2, March 2017.

[9] S. Davis, " Practical considerations with core geometry in inductor design and new products", APEC conference presentation, 2018.

[10] C. Xiao, G. Chen, W. G. H. Odendaal, "Overview of power loss measurement techniques in power electronics systems", IEEE Transactions on Industry Applications, Vol. 43 , No: 3 , May/ June 2017.

[11] Charles R. Sullivan,"Survey of core loss test methods", APEC conference presentation 2018.

[12] D. Christen, U. Badstuebner, J. Biela, and J.W. Kolar, " Calorimetric Power Loss Measurementfor Highly Efficient Converters", The International Power Electronics Conference, 2010.

[13] L.Aarniovuori, J. Kolehmainen, A, Kosonen, M, Niemelä, H. Chen, W, Cao, and Juha Pyrhönen, "Application of Calorimetric Method for Loss Measurement of a SynRM Drive System", IEEE Transactions on Industrial Electronics and Measurement, Vol. 63 , No: 4 , April 2016.

[14] V. Basso, M. Küpferling, C. P. Sasso, L. Guidici, "A Peltier cell calorimeter for the direct measurement of the isothermal entropy change in magnetic materials", Review of Scientific Instruments · July 2008.

[15] D. F. Frost, David A. Howey," High-Speed Peltier Calorimeter for the Calibration of High-Bandwidth Power Measurement Equipment", IEEE Transactions on Instrumentation and Measurement, vol. 65 , No. 1, January 2016.

[16] Y. Zhang, Thomas M. Jahns, "Power Electronics Loss Measurement Using New Heat Flux Sensor Based on Thermoelectric Device With Active Control", IEEE Transactions on Industry Applications, Vol. 50 , No. 6 , November/ December 2014

[17] M. Kuepferling, C. P. Sasso, V. B., and L. Giudici, "An Isothermal Peltier Cell Calorimeter For Measuring the Magnetocaloric Effect", IEEE Transactions on Magnetics, VOol. 43, No. 6, JUNE 2007.

[18] Y. Liu, L.Shi, Y. Li, "A calorimetry-based measurement apparatus for switching losses in high power electronic devices" IEEE International Energy Conference (ENERGYCON), April 2016.

[19] Y. Liu, L.Shi, Y. Li, "Comparison Analysis of Loss Calculation Methods and Measurement Techniques in Power Electronics and Motor Systems" International Conference on Electrical Machines and Systems, Oct. 2013.

[20] C. A. Baguley, U. K. Madawala1, and B. Carsten, "he Influence of Temperature and Core Geometry on Ferrite Core Losses under DC Bias Conditions", International Symposium on Power Electronics, Electrical Drives, Automation and Motion 2008.

[21] Planar ER cores and accessories ER32/6/25, Ferroxcube https://www.ferroxcube.com/upload/media/product/file/Pr_ds/ER32_6_ 25.pdf

[22] Thermoelectric cooler TEC1-12706: HB corporation, http://www.hebeiltd.com.cn/peltier.datasheet/TEC1-12706.pdf. 